\title{
Contagem de coliformes totais e Escherichia coli em alimentos no comércio ambulante: uma revisão integrativa
}

\section{Total coliform count and Escherichia coli in street food: an integrative review}

\author{
1 Ashley Brito Valentim ashley.valentim@aluno.uece.br \\ 2 Gissely Maria Ribeiro de Souza \\ 1 Gabrielly Rossi Monteiro \\ 3 Renata Carmo de Assis
}

\author{
Graduanda em Nutrição. Universidade Estadual do Ceará \\ Graduada em Nutrição. Universidade Estadual do Ceará \\ Mestre em Nutrição e Saúde. Especialista em Gestão da Qualidade em Serviço de Alimentação. Universidade Estadual do Ceará
}

\section{Resumo}

O consumo de comida vendida na rua é popular mundialmente. O objetivo desta pesquisa foi compilar dados que avaliaram as condições higiênico-sanitárias de alimentos vendidos em comércio ambulante, no ponto de vista de contagem de Coliformes totais e Escherichia coli. Para isso, a metodologia consistiu em uma revisão integrativa, seguindo etapas básicas: 1) elaborar o tema do estudo; 2) realizar a pesquisa bibliográfica; 3 ) organizar os dados coletados; 4) analisar os estudos incluídos na revisão; 5) interpretar e discutir os resultados; 6) sintetizar e divulgar a revisão. Foram selecionados 26 artigos para a revisão. Foi observado que a maioria dos alimentos comercializados em estabelecimentos ambulantes apresentaram resultados com níveis microbiológicos elevados. Concluiu-se que os alimentos vendidos na rua possuem níveis elevados de contaminação microbiológica.

\section{Palavras-chave:}

Colimetria. Microbiologia dos Alimentos. Serviços de Alimentação.

\begin{abstract}
The consume of food sold in the street is popular worldwide. The objective of this research was to compile data that evaluated the hygienic-sanitary conditions of food sold in street trade, from the point of view of counting total Coliforms and Escherichia coli. For this, the methodology consisted of an integrative review following basic steps: 1) elaborate the theme of the study; 2) carry out the bibliographic research; 3 ) organize the collected data; 4) analyze the studies included in the review; 5) interpret and discuss the results; 6) synthesize and disseminate the review. 26 articles were selected for the review. It was observed that the great majority of the food sold in ambulant establishments presented results with high microbiological levels. It was concluded that street food has high levels of microbiological contamination.
\end{abstract}

\section{Keywords:}

Colimetry. Food Microbiology. Food Services.

Como você deve citar?

VALENTIM, Ashley Brito et al. Contagem de coliformes totais e Escherichia coli em alimentos no comércio ambulante: uma revisão integrativa Cadernos UniFOA, Volta Redonda (RJ), v. 16, n. 47, p. 129 - 137, dez, 2021. 
O consumo de comida vendida na rua é popular devido à acessibilidade e disponibilidade (ASIEGBU et al., 2016). 0 maior percentual de consumo de alimentos fora do lar é proveniente de lanchonetes e restaurantes (BEZERRA et al., 2017). O comércio desses produtos é importante para a socioeconomia dos países em desenvolvimento, no entanto a natureza do comércio abre espaço para algumas atividades que podem representar sérios perigos para a saúde humana (ALIMI, 2016).

A ausência das práticas higiênicas é encontrada em vendedores ambulantes de alimentos. Assim, os consumidores devem ter conhecimento de higiene alimentar e critérios de escolha para os locais de aquisição dos alimentos, para minimizar o risco de intoxicação alimentar por microrganismos (TRAFIALEK et al., 2017).

Vendedores ambulantes de alimentos, em diferentes países, possuem baixo conhecimento sobre as Boas Práticas de Manipulação de Alimento e, consequentemente, sobre microorganismos patogênicos e doenças veiculadas por alimentos. As condições de manipulação e/ou processamento de alimentos também são precárias nesse tipo de comércio, sendo necessária a realização de ações de capacitação, conscientização e fiscalização sobre a manipulação adequada de alimentos (LIMA et al., 2017; ANDY et al., 2015).

A qualidade microbiológica dos alimentos em relação à vida de prateleira ou à segurança pode ser refletida através da presença de microrganismos indicadores. Dentre eles, os mais comuns são os patógenos provenientes de fontes intestinais, resultados de contaminação fecal, tais como Coliformes totais e Escherichia coli (JAY, 2006). Sabendo-se disso, objetivou-se compilar dados que avaliaram as condições higiênico-sanitárias de alimentos vendidos em comércio ambulante, no ponto de vista de contagem de Coliformes totais e Escherichia coli.

\section{MÉTODOS}

A revisão integrativa de literatura é baseada em pesquisas anteriores para, a partir delas, obter-se conclusões gerais. A pesquisa foi realizada em maio de 2019 e seguiu 6 (seis) etapas básicas, segundo Mendes et al. (2008).

$\mathrm{Na} 1^{\mathrm{a}}$ etapa, a pergunta norteadora envolve risco de Doenças Transmitidas por Alimentos (DTAs) advindas de alimentos comercializados na rua. Com isso, o que os estudos prévios apresentam de evidências sobre condições microbiológicas de contagem de Coliformes totais e $E$. coli em alimentos vendidos nas ruas?

$\mathrm{Na} 2^{\mathrm{a}}$ etapa, iniciou-se um levantamento bibliográfico com a utilização de artigos em português, inglês ou espanhol. Foram selecionados artigos disponibilizados no Periódicos da Coordenação de Aperfeiçoamento de Pessoal de Nível Superior (CAPES), Science Direct, Scielo, Lilacs, Google Acadêmico e nos repositórios de teses e dissertações da USP (Universidade de São Paulo) e da UNICAMP (Universidade de Campinas). Foi utilizada uma combinação de termos para uma busca avançada [Total Coliforms] AND [Escherichia coli] AND [street food sold in street vendors] AND [Foodborne Diseases]. Foram excluídos os estudos que não apresentavam foco na análise microbiológica de alimentos de rua, revisões e metanálises. Fez-se uma leitura exploratória dos títulos e resumos dos artigos, seguida de uma leitura para determinar se estavam adequados ao tema proposto.

Nas $3^{\mathrm{a}}$ e $4^{\mathrm{a}}$ etapas, os dados foram coletados segundo critérios de inclusão e exclusão, interpretados e avaliados, compilando-se as referências. 
Nas $5^{\mathrm{a}}$ e $6^{\mathrm{a}}$ etapas, os dados foram organizados para facilitar a análise crítica da amostra e as conclusões prévias obtidas a partir de cada leitura.

\section{RESULTADOS E DISCUSSÃO}

Na plataforma Periódicos Capes, foram encontrados 14 artigos; na base Science Direct foram encontrados 30 artigos; nas bases Scielo e Lilacs, nenhum artigo foi elegível; no Google Acadêmico, foram encontrados 2160 publicações diversas e nos repositórios da USP e UNICAMP foram encontradas 119 publicações, porém usando-se apenas Total Coliform como palavra-chave. Setenta artigos foram selecionados após leitura dos títulos e resumos, dos quais 41 artigos não atenderam ao critério de seleção. Após a aplicação dos critérios de exclusão e inclusão, restaram 26 artigos para a revisão final, os quais estão apresentados na tabela 1.

Percebeu-se publicações reduzidas em nível nacional referentes aos estudos que analisaram alimentos vendidos por ambulantes no Brasil. Tenta-se justificar a escassez dessas publicações no Brasil, pelo fato de que fenômenos pouco significativos em sociedades da América Latina raramente despertam a atenção dos pesquisadores (SOARES, 2005).

Outros fatores podem influenciar essa escassez, tais como, avaliação de qualidade questionável, processo de revisão muito longo, ausência de feedback para avaliação e, de forma geral, o processo da pesquisa, submissão e publicação requererem muito tempo e dedicação (SERRA et al., 2008).

Embora alguns estudos tenham analisado outros tipos de microrganismos, coletou-se os dados apenas de Coliformes totais e Escherichia coli dos artigos selecionados, pois por si só, são fortes indicadores de contaminação de origem fecal, podendo ser causadores de DTA (AMARAL et al., 2012).

Uma relevante dissertação apresentou diferentes olhares sobre o comércio de alimentos na Universidade de São Paulo/Brasil, constatando que, apesar de tudo, é possível a prática do comércio de comida de rua com qualidade higiênica-sanitária, sem caracterizar uma ameaça à saúde pública (OLIVEIRA, 2014).

Os Critérios Microbiológicos de Higiene $(\mathrm{CH})$ definem a aceitabilidade de um alimento em função da presença ou quantidade de microrganismos indicadores de higiene. Esses critérios são determinados de acordo com grupos de alimentos similares, que, em uma amostra representativa, podem variar em níveis aceitáveis de 2 a $5 \times 10$ UFC/g para Coliformes totais (BRASIL, 2001) e de 7 a $5 \times 10^{3} \mathrm{UFC/g}$ para Escherichia coli (BRASIL, 2019).

Diante do exposto na tabela 1, percebe-se que a maioria dos alimentos vendidos por ambulantes nas ruas apresentam níveis críticos de Coliformes Totais e $E$. Coli. Isso retrata que esses alimentos representam um risco à saúde pública em nível nacional e internacional. Elobeid et al. (2014) sugere que os governos poderiam fornecer apoio às pequenas empresas alimentares, ajudando na implementação de uma avaliação sistemática dos agentes patogênicos de origem alimentar em todas as organizações de serviços alimentares e todos os manipuladores de alimentos que trabalham em serviço alimentar e, consequentemente, na prevenção de patologias.

A partir da análise da tabela 1 constatou-se que, de todos os estudos, aproximadamente, $19 \%$ detinham o resultado não analisado (NA) e, aproximadamente, $4 \%$ não detectado (ND) para Coliformes Fecais em alimentos. Já para Escherichia coli em alimentos, aproximadamente, $54 \%$ dos artigos possuíam o resultado não analisado (NA) e, aproximadamente, 8\% não detectado (ND). 
Tabela 1 - Ordem cronológica decrescente dos 26 artigos selecionados para revisão bibliográfica final, segundo os critérios utilizados. Cada referência reúne um resumo dos dados extraídos pertinentes ao presente estudo.

\begin{tabular}{|c|c|c|c|c|}
\hline Referência & País & Alimento & $\begin{array}{l}\text { Contagem de Coliformes } \\
\text { Totais }\end{array}$ & Contagem de $E$. coli \\
\hline \multirow{3}{*}{ Oliveira et al., 2019} & \multirow{3}{*}{ Vitória (Brasil) } & Salgado & $<10^{1}-1,5 \times 10^{4}$ UFC/g & ND \\
\hline & & Água de coco & $9,6 \times 10^{2}-2,5 \times 10^{4}$ UFC/g & ND \\
\hline & & Cachorro quente & $2,3 \times 10^{4}-2,5 \times 10^{4}$ UFC/g & ND \\
\hline Djibrine et al., 2018 & N’Djamena (Chade) & Sanduíches & $(0,16-3,0) \times 10^{6} \mathrm{UFC} / \mathrm{g}$ & $(0,00-0,40) \times 10^{6} \mathrm{UFC} / \mathrm{g}$ \\
\hline Happy et al., 2018 & Dakha (Bangladesh) & Chotpoti & $6 \times 10^{5}$ (1 local) UFC/g & NA \\
\hline Hassan et al., 2017 & Dakha (Bangladesh) & Chotpoti & ND & $2,8 \%$ \\
\hline $\begin{array}{l}\text { Cerna-Cortes et al., } \\
2016\end{array}$ & México & Suco de laranja & $73 \mathrm{NMP} / \mathrm{mL}$ & $<3 \mathrm{NMP} / \mathrm{mL}$ \\
\hline Kothe et al., 2016 & Porto Alegre (Brasil) & Cachorro-quente & $\begin{array}{l}2,3 \times 10^{-1 /} />1,1 \times 10^{3} \\
\mathrm{NMP} / \mathrm{g}(16 \text { locais })\end{array}$ & NA \\
\hline \multirow{4}{*}{ Bereda et al., 2016} & \multirow{4}{*}{ Jigjiga (Etiópia) } & $\begin{array}{l}\text { Fuol (feijão c/ } \\
\text { especiarias.) }\end{array}$ & $3.6 \times 10^{4} \mathrm{UFC} / \mathrm{g}$ & \multirow{4}{*}{ NA } \\
\hline & & Pastel c/ lentilha & & \\
\hline & & Massa & $7,3 \times 10^{4} \mathrm{UFC} / \mathrm{g}$ & \\
\hline & & Leite de camelo & $4,3 \times 10^{4} \mathrm{UFC} / \mathrm{g}$ & \\
\hline \multirow{4}{*}{ Ike et al., 2015} & \multirow{4}{*}{ Abia (Nigéria) } & Torta de carne & $(1,7-2,6) \times 10^{2} \mathrm{UFC} / \mathrm{g}$ & NA \\
\hline & & Torta de peixe & $1,7 \times 10^{1}-2,2 \times 10^{2}$ UFC/g & NA \\
\hline & & Chip de banana & $(0.5-1,0) \times 10^{1} \mathrm{UFC} / \mathrm{g}$ & NA \\
\hline & & Batata frita & $1,3 \times 10^{1}-1,4 \times 10^{2}$ UFC/g & NA \\
\hline \multirow{5}{*}{ Amin et al., 2015} & \multirow{5}{*}{ Dhaka (Bangladesh) } & Arroz tufado & $2,32-2,42 \log _{10} \mathrm{UFC} / \mathrm{g}$ & $2,02-2,08 \log _{10} \mathrm{UFC} / \mathrm{g}$ \\
\hline & & Bolo & $1,88-2,31 \log _{10} \mathrm{UFC} / \mathrm{g}$ & $1,57-1,92 \log _{10} \mathrm{UFC} / \mathrm{g}$ \\
\hline & & Grão de bico & $2,32-2,89 \log _{10} \mathrm{UFC} / \mathrm{g}$ & $1,87-2,64 \log _{10} \mathrm{UFC} / \mathrm{g}$ \\
\hline & & Pastel & $1,77-2,45 \log _{10} \mathrm{UFC} / \mathrm{g}$ & $1,57-1,94 \log _{10} \mathrm{UFC} / \mathrm{g}$ \\
\hline & & Caldo de cana & $2,76-3,54 \mathrm{Log}_{10} \mathrm{UFC} / \mathrm{g}$ & $2,46-3,39 \log _{10} \mathrm{UFC} / \mathrm{g}$ \\
\hline Elobeid et al. 2014 & Qatar & $\begin{array}{l}\text { Alimentos } \\
\text { tradicionais }\end{array}$ & NA & $3 \%$ \\
\hline Khandoker et al., 2014 & Bangladesh & Arroz tufado picante & $(1,4-4,3) \log$ UFC/g & NA \\
\hline Oliveira, 2014 & São Paulo (Brasil) & Salgados e doces & $2,1 \times 10^{7} \mathrm{UFC} / \mathrm{g}$ & $4,4 \times 10^{6} \mathrm{UFC} / \mathrm{g}$ \\
\hline $\begin{array}{l}\text { Tabashsum et al., } \\
2013\end{array}$ & Dakha (Bangladesh) & 13 Comidas típicas & $2,0-7,5$ log UFC/g & NA \\
\hline Min et al., 2013 & $\begin{array}{l}\text { Canterbury (Nova } \\
\text { Zelândia) }\end{array}$ & Hambúrguer & $(1,50-2,25) \times 10^{2} \mathrm{UFC} / \mathrm{g}$ & ND \\
\hline \multirow{2}{*}{ Mamun et al., 2013} & \multirow{2}{*}{ Dakha (Bangladesh) } & Chotpoti & \multirow{2}{*}{ NA } & NA \\
\hline & & Arroz tufado & & NA \\
\hline Derbew et al., 2013 & Gongar (Etiópia) & Comidas típicas & $(1,3-4,6) \times 10^{3} \mathrm{UFC} / \mathrm{g}$ & NA \\
\hline \multirow{4}{*}{$\begin{array}{l}\text { Mugampoza et al., } \\
2013\end{array}$} & \multirow{4}{*}{ Nakawa (Uganda) } & Caldo de carne & NA & $0,29 \log _{10} \mathrm{UFC} / \mathrm{g}$ \\
\hline & & Ensopado de peixe & NA & $0,29-0,36 \log _{10} \mathrm{UFC} / \mathrm{g}$ \\
\hline & & Ovo frito & NA & $4,27-7,48 \log _{10} \mathrm{UFC} / \mathrm{g}$ \\
\hline & & Salada & NA & $0,28-11,99 \log _{10} \mathrm{UFC} / \mathrm{g}$ \\
\hline Samuel, 2012 & Delta (Nigéria) & 106 Pratos típicos & 36 a $2100 \mathrm{NMP} / \mathrm{g}$ & NA \\
\hline Ates et al., 2011 & Ankara & Mexilhão recheado & NA & $3,5 \times 10^{2}$ \\
\hline Tambekar et al., 2011 & Amravati (Índia) & Panipuri (Purê frito) & NA & NA \\
\hline Ukwo et al., 2011 & Uyo (Nigéria) & Suco de frutas & 3,30-4,53 log UFC/100mL & NA \\
\hline
\end{tabular}




\begin{tabular}{|c|c|c|c|c|}
\hline \multirow{4}{*}{ Cho et al., 2011} & \multirow{4}{*}{ Korea } & Suco de fruta & \multirow{4}{*}{$3,24( \pm 1,4) \log _{10}$ UFC/g } & \multirow{4}{*}{$2,33( \pm 0,90) \log$ UFC/g } \\
\hline & & Sanduíche & & \\
\hline & & Comida frita & & \\
\hline & & Kim-bab (Sushi) & & \\
\hline Curi, 2006 & São Paulo (Brasil) & Hot dog & $\begin{array}{l}1,5 \times 10^{2} \text { a } 11 \times 10^{4} \\
\text { NMP } / g\end{array}$ & NA \\
\hline Hanashiro et al., 2005 & São Paulo (Brasil) & $\begin{array}{l}\text { Sanduíche quentes } \\
\text { e frios }\end{array}$ & $>2,4-10^{4} \mathrm{NMP} / \mathrm{g}$ & NA \\
\hline Fattori, 2003 & São Paulo (Brasil) & X-salada & $\begin{array}{l}2,25 \times 10^{2} \text { até } 16 \times 10^{3} \\
\text { UFC/g }\end{array}$ & NA \\
\hline Rodrigues et al., 2003 & $\begin{array}{l}\text { Rio Grande do Sul } \\
\text { (Brasil) }\end{array}$ & Cachorro quente & $10^{5}$ a $10^{6} \mathrm{UFC} / \mathrm{g}$ & NA \\
\hline
\end{tabular}

E. coli (Escherichia coli); UFC (Unidade Formadora de Colônias); g (gramas); NMP (Número Mais Provável); NA (Não Analisado); ND (Não Detectado).

A análise realizada em uma cidade brasileira para Escherichia coli nos alimentos, em que se constata como resultado o não detectado (ND), ocorreu por não se encontrar amostras desse microorganismos no alimento. (OLIVEIRA, et al., 2019). Para os demais estudos que obtiveram o resultado não aplicado (NA), é justificado pela não ocorrência da análise desse microrganismo.

O consumo de alimentos vendidos por ambulantes não deve ser interrompido, assim como os vendedores de rua não devem ser proibidos de vender tais itens, já que essa é uma fonte de renda para seu sustento (OLIVEIRA; ARAÚJO; SILVA, 2013; DE OLIVEIRA et al., 2010; LEONE, 2010).

No entanto, o estado pode trabalhar na orientação e formalização dos ambulantes, seja através de incentivos fiscais, no maior rigor da fiscalização ou da institucionalização de pontos específicos dentro do espaço das cidades (ROSA, 2014).

Os órgãos fiscais de saúde podem realizar capacitações dos vendedores sobre Boas Práticas de Fabricação (BPF), Boas Práticas de Higiene (BPH) e Doenças Transmitidas por Alimentos (DTA), a fim de se garantir a segurança dos alimentos comercializados, podendo-se exigir a declaração de participação nessas capacitações como um dos documentos necessários para a finalização do licenciamento (DE SOUSA; AGUIAR, 2019).

Tais fiscalizações, nos estudos analisados, pelos órgãos competentes precisam ser rigorosas e constantes (HAPPY et al., 2018; DERBEW et al., 2014; MUGAMPOZA et al., 2013; UKWO et al., 2011), inclusive com exigência de licença sanitária e de condições ambientais adequadas (OLIVEIRA et al., 2019).

Higiene pessoal e manuseio do alimento inadequado, práticas de transporte e armazenamento de alimentos e pouco conhecimento dos vendedores em relação à DTA são alguns dos fatores de riscos associados à contaminação de alimentos vendidos na rua (OLIVEIRA et al., 2019; HAPPY et al., 2018; DERBEW et al., 2014; KOTHE et al., 2016; TAMBEKAR et al., 2011).

É necessário supervisão mais frequente na inspeção por vigilância sanitária para reforçar a aplicabilidade de medidas de controle de segurança, como, por exemplo, o controle de tempo e temperatura, o uso de água potável, a prevenção de contaminação cruzada e uso de matéria-prima com origem identificada (KOTHE et al., 2016; ELOBEID et al., 2014, MAMUN et al., 2013).

O estado de São Paulo possui uma legislação própria em nível estadual, que é a Resolução SS n 142/93 (Centro de Vigilância Sanitária, 1993), que estabelece as normas técnicas para o comércio ambulante de alimentos. E, em estados brasileiros que são carentes de legislações locais, é aconselhá- 
vel o uso da Resolução Diretoria Colegiado RDC 216 (BRASIL, 2004), legislação que orienta a conduta higiênico-sanitária para comida de rua comercializada por vendedores ambulantes ou, ainda, pode se fazer uma adaptação à resolução de São Paulo.

\section{CONSIDERAÇÕES FINAIS}

Os alimentos vendidos nas ruas possuem um elevado nível de contaminação por Coliformes totais e Escherichia coli. Os vendedores ambulantes que manipulam e vendem alimentos não realizam "Boas Práticas de Fabricação", segundo a legislação nacional ou estadual vigente no país (BRASIL, 2004; CENTRO DE VIGILÂNCIA SANITÁRIA, 1993), fornecendo assim, alimentos com risco à saúde pública.

A fiscalização por parte dos órgãos públicos nacionais é deficiente (BOANOVA, 2014) e um amplo estudo realizado no Brasil, por Kothe et al. (2016), cita a real necessidade de fiscalizações mais frequentes e rígidas por setores competentes, bem como Happy et al. (2018) e Derbew et al. (2014), que, em nível internacional, também fortalecem essa ideia. A regulamentação desse setor da economia, junto à criação de campanhas de conscientização e educação seriam importantes para minimizar essa problemática.

\section{REFERÊNCIAS}

ALIMI, B.A. Risk factors in street food practices in developing countries: A review. Food Science and Human Wellness, Beijing, v. 5, n. 3, p. 141-148, 2016.

AMARAL, D. A.; GREGÓRIO, E. L.; SILVA, M.; OLIVEIRA, J. H. M.; BASTOS, B. F. M. Análise microbiológica do acarajé comercializado numa feira de arte e artesanato de Belo Horizonte, MG. HU Revista, v. 38, p. 175-180, 2012.

ANDY, E.; MANGAI, J. M.; KAYONG; E. A.; AFOI, B. B.; GOSHIT, J. D.; KASANG, N. et al. Assessment of Practice of Food Safety and Hygiene among Food Vendors within Jos North Local Government Area of Plateau State, Nigeria. International Journal of Medical and Health Research, v. 1, n. 2, p. 83-6, 2015.

ASIEGBU, C. V.; LEBELO, S. L.; TABIT, F. T. The food safety knowledge and microbial hazards awareness of consumers of ready-to-eat street-vended food. Food Control, v. 60, p. 422-429, 2016.

ATES, M.; OZKIZILCIK, A.; TABAKOGLU, C. Microbiological Analysis of Stuffed Mussels Sold in the Streets. Indian Journal of Microbiology, v. 51, p. 350-354, 2011.

BEREDA, T. W.; EMERIE, Y. M.; RETA, M. A.; ASFAW, H. S. Microbiological Safety of Street Vended Foods in Jigjiga City, Eastern Ethiopia. Ethiopian Journal of Health Science, v. 26, n. 2, p.161-70, 2016.

BEZERRA, I. N.; MOREIRA, T. M. V; CAVALCANTE, J. B.; SOUZA, A. M.; SICHIERIL, R. Consumo de alimentos fora do lar no Brasil segundo locais de aquisição. Revista de Saúde Pública, v. 51, p. 1-8, 2017.

BOANOVA, A. B. Análises e interpretação de denúncias sobre alimentos como ferramenta de gestão no município de São Paulo. Doutorado. Programa de pós-graduação em saúde pública. Universidade de São Paulo. 2014. 126p.

BRASIL. Resolução RDC N 12, de 2 de janeiro de 2001. Regulamento técnico sobre padrões microbiológicos para alimentos. Órgão emissor: ANVISA - Agência Nacional da Vigilância Sanitária, 2001. 
BRASIL. Ministério da Saúde. Secretaria de Vigilância Sanitária. Agência Nacional de Vigilância Sanitária. Resolução RDC n. 216, 15 de setembro de 2004. Dispõe sobre regulamento técnico de boas práticas para serviços de alimentação.

BRASIL. Ministério da Saúde. Agência Nacional de Vigilância Sanitária. Instrução Normativa ${ }^{\circ}$ 60, de 23 de dezembro de 2019. Estabelece as listas de padrões microbiológicos para alimentos. 2019.

CENTRO DE VIGILÂNCIA SANITÁRIA. Resolução SS-142, de 03 de maio de 1993. Aprova Norma Técnica Relativa ao Comércio Ambulante de Gêneros Alimentícios. Diário Oficial Estadual, São Paulo, SP, 04 de maio de 1993.

CERNA-CORTES, J. F.; CORTES-CUERTO, A. N. A. L.; CANO-GAONA, M. R., LEON-MONTES, N.; HELGUERAREPETTO, A. C.; RIVERA-GUTIERREZ, S. et al. Microbiological Quality and Occurrence of Nontuberculous Mycobacteria in Fresh-Squeezed Orange Juice Samples Purchased from Street Vendors in Mexico City. Journal of Food Protection, v. 79, p. 2190-2195, 2016.

CHO, J.; CHEUNG, C.; LEE, S.; KO, S.; KIM, K.; HWANG, I.; KIM, S.; CHO, S.; LIM, C.; LEE, K.; KIM, K. Assessment of microbial contamination levels of street vended foods in Korea. Journal Food Safety, v. 31, p. 41-7, 2011.

CURI, J. D. P. Condições Microbiológicas de lanches (cachorro quente) adquiridos de vendedores ambulantes, localizadas na parte central da cidade de Limeira - SP. Dissertação. Universidade de São Paulo. Escola Superior de Agricultura "Luiz de Queiroz" 2014. 110p.

DE OLIVEIRA, G. F.; CARREIRO, G. S. P.; FILHA, M. D. O. F.; LAZARTE, R.; DE TOLEDO VIANNA, R. P. Risco para depressão, ansiedade e alcoolismo entre trabalhadores informais. Revista Eletrônica de Enfermagem, v. 12, n. 2, p. 272-7, 2010.

DERBEW, G.; SAHLE, S.; ENDRIS, M. Bacteriological Assessment of Some Street Vended Foods in Gondar, Ethiopia. Internet J Food Safety, v. 15, p. 33-38, 2014.

DE SOUSA, M. G. K.; AGUIAR, L. P. A VIGILÂNCIA SANITÁRIA E O COMÉRCIO DE ALIMENTOS EM EVENTOS DE MASSA. Cadernos ESP-Revista Científica da Escola de Saúde Pública do Ceará, v. 13, n. 2, p. 38-53, 2019.

DJIBRINE, M. A.; TIDJANI, A.; NGANDOLO, B. N.; NADLAOU, B.; BARRO, N. Microbiological quality of some street foods in N'Djamena, Chad: case of sandwiches. International Journal of Biological and Chemical Science, v. 12, n.3, p. 1113-1122, 2018.

ELOBEID, T.; AZIZ, H.A.; MOUSA, R. Survey on the Microbial Quality of Traditional Foods Sold by Street Vendors in Qatar. Austin Journal of Nutrition and Metabolism, v. 1, p. 2-5, 2014.

FATTORI, F. F. A. Aspectos Sanitários em Trailers de Lanche do Município de Presidente Prudente-SP. Dissertação. Programa de Pós-Graduação de Medicina Veterinária da Faculdade de Medicina Veterinária e Zootecnia de Botucatu, Universidade Estadual Paulista. São Paulo. 2003. 94p.

HANASHIRO, A.; MORITA, M.; MATTÉ, G. R.; MATTÉ, M. H.; TORRES, E. A. F. S. Microbiological quality of selected street foods from a restricted area of São Paulo city, Brazil. Food Control, v. 16, n. 5, p. 439-444, 2005 . 
HAPPY, A. H.; ALAM, M. G., MAHMUD, S.; IMRAN, M. A. S.; RONY, M. H., AZIM, M. A. A. et al. Isolation, Identification and Characterization of Gram Negative Bacteria from Popular Street Food (Chotpoti) at Savar Area, Dhaka. Open Access Library Journal, v. 5, n. 11, p. 1-11, 2018.

HASSAN, M. Z.; ISLAM, M. S.; SALAUDDIN, M.; ZAFOR, A. H. A.; SCOTT, M. L., ALAM, S. Detection of enteric bacteria in the popular street food chotpoti in Dhaka,. Asian Journal of Medical and Biological Research, v. 2, n.4, p. 596-602, 2017.

IKE, C. C.; EMEKA-IKE, PC, NWOKORIE, C. C.; ANOCHIE, C. C. Microbiological quality evaluation of locally prepared snacks sold in Aba metropolis, Abia State, Nigeria. International Journal of Science Engineering and Applied Sciences, v. 1, n. 7, p. 2395-3470, 2015.

OLIVEIRA, J. M.; ARAÚJO, B. C. P. O.; SILVA, L. V. Panorama da economia criativa no Brasil. 2013.

JAY, J. M. Microbiologia de Alimentos. 6. ed. Porto Alegre: Artmed, 2006.

KHANDOKER, A.; ISLAM, M. A.; RAHMAN, M. M.; HUSNA, A. A.; DAS, S.; KHATUN, M. M. Bacterial contamination of street-vended spicy puffed-rice sold at Bangladesh Agricultural University campus. Bangladesh Vet, v. 31, n. 1, p. 20-26, 2014

KOTHE, C.I.; SCHILD, C. H.; TONDO, E. C.; MALHEIROS, P.S. Microbiological contamination and evaluation of sanitary conditions of hot dog street vendors in Southern Brazil. Food Control, v. 62, p. 364-350, 2016.

LEONE, EUGENIA TRONCOSO. O perfil dos trabalhadores e trabalhadoras na economia informal. ILO, 2010.

LIMA, T. S.; JESUS, I. G. D.; SANT'ANNA, M. D. S. L.; FEITOSA, P. R. B. Condições higienicossanitárias de alimentos comercializados por ambulantes no centro comercial de Aracaju, SE. Higiene Alimentar, v. 31, n. 270/271, 2017.

MAMUN, M. A.; RAHMAN, S. M. M.; TURIM, T. C. Microbiological quality of selected street food items vended by school-based street food vendors in Dhaka, Bangladesh. International Journal of Food Microbiology, v. 166, p. 413-418, 2013.

MENDES, K. D. S.; SILVEIRA, R. C. C. P.; GALVÃO, C. M. Revisão Integrativa: método de pesquisa para a incorporação de evidências na saúde e na enfermagem. Texto \& Contexto - Enfermagem, v. 17, n. 4, p. 758-764, 2008.

MIN, M.; DAWSON, C. O.; HUSSAIN, M. A. Microbiological Risk Assessment of Hamburgers Sold in Canterbury New Zealand. Internet Journal of Food Safety, v. 13, p. 99-102, 2013.

MUGAMPOZA, D.; BYARUGABA, G. W. B., NYONYINTONO, A.; NAKITTO, P.; CAMPUS, B.; KINGDOM, U. Occurrence of Escherichia coli and Salmonella spp . in street-vended foods and general hygienic and trading practices in Nakawa Division, Uganda. American Journal of Food and Nutrition, v. 3, n. 3, p. 167175, 2013.

OLIVEIRA, JOÃO MARIA DE; ARAÚJO, BRUNO CÉSAR PINO OLIVEIRA DE; SILVA, LEANDRO VALÉRIO. Panorama da economia criativa no Brasil. Instituto de Pesquisa Econômica Aplicada.- Brasília : Rio de Janeiro : Ipea , 1990- 2013. 
OLIVEIRA, J. S. C.; JOSÉ, J. F. B. S. Food Handling Practices and Microbial Quality in Street Food. Journal of Food and Nutrition Research, v. 7, p. 319-324, 2019.

OLIVEIRA, T. H. N. Diferentes olhares sobre o comércio de alimentos na Universidade de São Paulo/ Brasil. Dissertação Mestrado - Universidade de São Paulo. Faculdade de Medicina Veterinária e Zootecnia. Departamento de Medicina Veterinária Preventiva e Saúde Animal, São Paulo, 2014. 104p.

RODRIGUES, K. L.; GOMES, J. P. ; CONCEIÇÃO, R. C. S.; BROD, C. S.; CARVALHAL, J. B.; ALEIXO, J. A. G. Condições higiênico-sanitários no comércio ambulante de alimentos de Pelotas-RS. Revista Ciências e Tecnologia de Alimentos. v. 23, n. 3, p. 447-452, 2003.

ROSA, T. A. 0 Comércio Ambulante de Alimentos em Uberlândia - MG. 2014. 134 f. Monografia (Bacharelado em Geografia) - Instituto de Geografia, Universidade Federal de Uberlândia, Uberlândia, 2014.

SAMUEL, O. O. Bacteriological Quality and safety of Street Vended Foods in Delta State, Nigeria. Journal of Biology, Agriculture and Healthcare, v. 2, p. 2008-2013, 2012.

SÃO PAULO (Município). Secretaria Municipal de Saúde. SMS-G n 2.619/11. Aprova o regulamento de boas práticas e de controle condições sanitárias e técnicas das atividades relacionadas (São Paulo, 2011)

SERRA, F. A. R.; FIATES, G. G.; FERREIRA, M. P. Publicar é difícil ou faltam competências? O desafio de pesquisar e publicar em revistas científicas na visão de editores e revisores internacionais. RAM, Revista de Administração Mackenzie. v. 9, n. 4, p. 32-55, 2008.

SOARES, G. A. D. O Calcanhar Metodológico da Ciência Política no Brasil. Sociologia, Problemas e Práticas, n. 48, p. 27-52, 2005.

TABASHSUM, Z.; KHALIL, I.; BARI, L. Prevalence of Foodborne Pathogens and Spoilage Microorganisms and Their Drug Resistant Status in Different Street Foods of Dhaka city. Agriculture Food and Analytical Bacteriology, v. 3, n. 4, p. 281-292, 2013.

TAMBEKAR, D. H.; KULKARNI, R. V.; SHIRSAT, S. D.;BHADANGE, D. G. Bacteriological quality of street vended food panipuri : a case study of Amravati city (ms) India. Bioscience Discovery, v. 2, p. 450-354, 2011.

TRAFIALEK, J.; DROSINOS, E. H., LASKOWSKI, W.; JAKUBOWSKA-GAWLIK, K.; TZAMALIS, P.; LEKSAWASDI, N. et al. Street food vendors' hygienic practices in some Asian and EU countries - A survey. Food Control, v. 85, p. 212-222, 2017.

UKWO, S. P.; NDAEYO, N. U.; UDOH, E. J. Microbiological Quality and Safety Evaluation of Fresh Juices and Edible Ice Sold in Uyo Metropolis, South-South, Nigeria. Internet Journal of Food Safety, v. 13, p. 374-8, 2011. 